\title{
Inflammation and the Peritoneal Membrane: Causes and Impact on Structure and Function during Peritoneal Dialysis
}

\author{
Gilberto Baroni, Adriana Schuinski, Thyago P. de Moraes, \\ Fernando Meyer, and Roberto Pecoits-Filho
} School of Medicine, Pontifical Catholic University of Paraná, State University of Ponta Grossa,
80215-901 Curitiba, PR, Brazil

Correspondence should be addressed to Roberto Pecoits-Filho, r.pecoits@pucpr.br

Received 14 November 2011; Accepted 16 January 2012

Academic Editor: Markus Wörnle

Copyright ( $\odot 2012$ Gilberto Baroni et al. This is an open access article distributed under the Creative Commons Attribution License, which permits unrestricted use, distribution, and reproduction in any medium, provided the original work is properly cited.

Peritoneal dialysis therapy has increased in popularity since the end of the 1970s. This method provides a patient survival rate equivalent to hemodialysis and better preservation of residual renal function. However, technique failure by peritonitis, and ultrafiltration failure, which is a multifactorial complication that can affect up to $40 \%$ of patients after 3 years of therapy. Encapsulant peritoneal sclerosis is an extreme and potentially fatal manifestation. Causes of inflammation in peritoneal dialysis range from traditional factors to those related to chronic kidney disease per se, as well as from the peritoneal dialysis treatment, including the peritoneal dialysis catheter, dialysis solution, and infectious peritonitis. Peritoneal inflammation generated causes significant structural alterations including: thickening and cubic transformation of mesothelial cells, fibrin deposition, fibrous capsule formation, perivascular bleeding, and interstitial fibrosis. Structural alterations of the peritoneal membrane described above result in clinical and functional changes. One of these clinical manifestations is ultrafiltration failure and can occur in up to $30 \%$ of patients on PD after five years of treatment. An understanding of the mechanisms involved in peritoneal inflammation is fundamental to improve patient survival and provide a better quality of life.

\section{Introduction}

Peritoneal dialysis (PD) therapy has increased in popularity since the end of the 1970s. The method was developed as an alternative to hemodialysis (HD) presenting a patient survival rate equivalent to $\mathrm{HD}$ and better preservation of residual renal function. However, technique failure remains high, resulting in frequent modality changes. Currently, the two principal causes of technique failure in order of importance are (a) peritonitis, this important medical problem can also represent nearly $16 \%$ of the causes of death; (b) ultrafiltration failure, a multifactorial complication that can affect up to $40 \%$ of patients after 3 years of therapy [1].

The peritoneal membrane is composed of different cell types with varying functions. Peritonitis as well as contact with bioincompatible solutions have deleterious effects on the membrane. These proinflammatory stimuli can induce lymphokine secretion by macrophages, which in turn, activate fibroblasts. Fibroblast activation has been associated with structural alterations in the peritoneal membrane of varying intensity. These alterations can be seen in Figure 1 which was extracted from a submitted study of our group. In this prospective controlled study in 20 nonuremic Wistar rats, peritoneal fibrosis occurs after exposure to glucosebased PD solutions and regardless the use of simvastatin.

Encapsulant peritoneal sclerosis (EPS) is an extreme and potentially fatal manifestation. EPS is a clinical syndrome that leads to persistent or recurrent intestinal obstruction, with or without inflammatory parameters of peritoneal thickening, sclerosis, calcification, and encapsulation, and can be inferred by clinical symptoms and radiology, but confirmed only by direct visualization with laparotomy $[2,3]$. Incidence of EPS is heterogenous and has been 

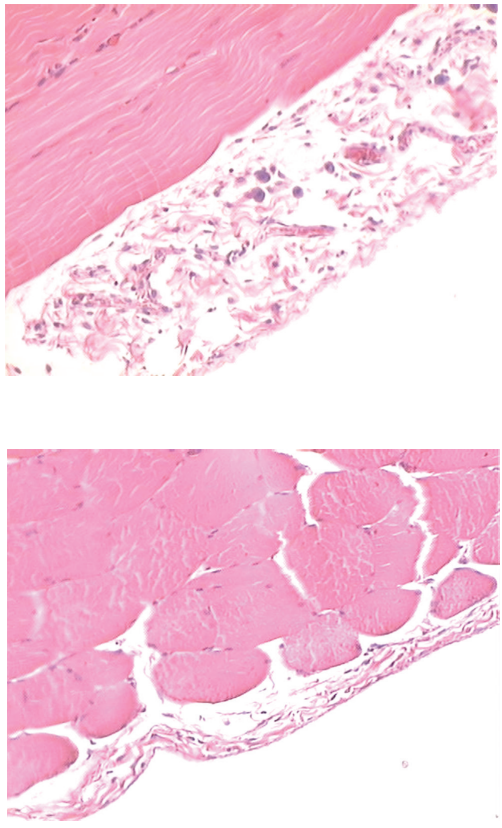
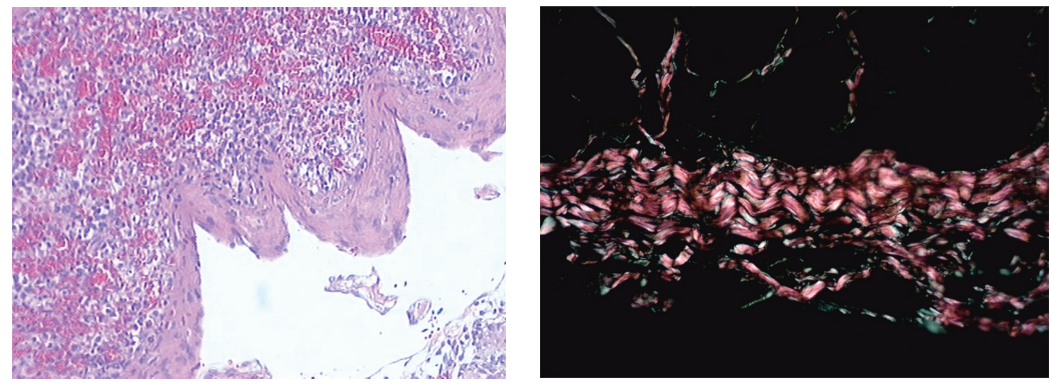

(a)
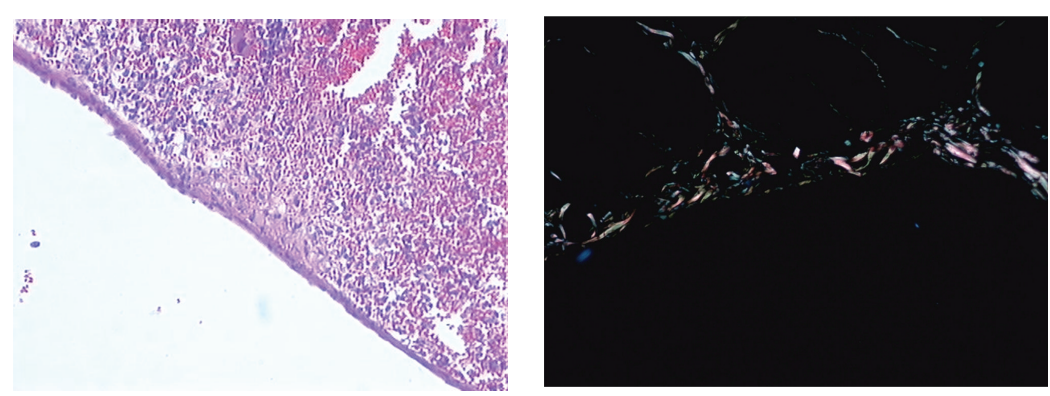

(b)

Figure 1: Typical alterations in the peritoneal membrane in an experimental model of hypertonic dialysate infusion (a) and the impact of oral statin use during 8 weeks of followup (b).

reported to vary from 6 to $20 \%$ in eight years depending on the region.

\section{Causes of Inflammation in PD}

Causes of inflammation in peritoneal dialysis range from traditional factors to those related to chronic kidney disease per se as well as from the peritoneal dialysis treatment itself.

Uremia is a factor present in all PD patients and generates an inflammatory state causing stress on the peritoneum due to the formation of carbonyl products. It accelerates the formation of advanced glycation end products (AGEs) that induces an upregulation of the receptors of advanced glycation end products (RAGE) [4]. Dialysis decreases the impact of uremia, however, does not remove it completely.

The peritoneal dialysis catheter is the first proinflammatory factor associated to $\mathrm{PD}$ with which the patient comes into contact. After implantation in the peritoneum, the catheter can induce an inflammatory reaction as was demonstrated by Flessner et al. [5]. In addition, the catheter can occasionally be the site of bacterial biofilm formation.

Initial therapy introduces the second inflammatory factor associated with PD: dialysis solution. Several PD solutions are available on the market today, and all are, to varying degrees, associated with peritoneal inflammation. Such inflammation is generated by several characteristics of these solutions, varying from low $\mathrm{pH}$, presence of lactate, hyperosmolality, increased glucose concentration, presence of glucose degradation products (GDP) and advanced glycation end products (AGEs), and icodextrin metabolites, among others $[6,7]$.
Currently available glucose-based PD solutions present concentrations varying between 1.5 and $4.25 \%$ of glucose. The glucose load offered daily by a traditional PD prescription usually ranges from $120 \mathrm{~g}$ to $400 \mathrm{~g}$.

The majority of PD solutions prescribed today markedly acidify $\mathrm{pH}$ to nearly 5.7 in approximately 2 to 3 minutes. This $\mathrm{pH}$ decreases viability of neutrophils and mesothelial cells, thus decreasing cytokine production and phagocytosis cap-acity. Lactate is utilized as a buffer in the majority of solutions. Its bioincompatibility with the peritoneal membrane is well known as well as its capacity to stimulate the production of fibroblast growth factors contributing to peritoneal fibrosis [8].

The association of icodextrin with EPS development is controversial. Some studies have associated the osmotic agent with EPS development [7], while others have shown it to be distinct, confirming its safety even with long-term utilization [6]. The relative rarity of the disease makes a definitive conclusion difficult. Even experimental studies with rats addressing this question are compromised by the increased $\alpha$-amylase activity in these animals. The presence of this enzyme in plasma and in the peritoneal cavity provokes a rapid drop in peritoneal icodextrin concentration [9].

Chronic exposure to high glucose load in traditional PD solution induces significant inflammation of the peritoneal membrane. These solutions induce several proinflammatory factors such as PGA [10], vascular endothelial growth factors (VEGFs), fibroblast growth factor (TGF- $\beta 1$ ), AGEs, and upregulation of RAGEs. Together, these factors contribute to the occurrence of neoangiogenesis and mesothelial fibrosis [11]. Glucose degradation products (GDPs), such 
as methylglyoxal, glyoxal, and 3-deoxyglucosone generated during the heat sterilization process, increase inflammation by inducing oxidative stress, which thus causes damage to mesothelial cells and leads to apoptosis and mesothelial denudation [12].

Substituting traditional solutions for more biocompatible solutions was recently associated with reduced membrane alterations [13]. It has been suggested for some years that the pathway of transforming growth factor $\beta 1 /$ Smad plays a part in the development of peritoneal fibrosis. High glucose concentration in PD solutions is related to the activation of this pathway. The relationship between Smad2 and VEGF expression has also been reported. The latter is recognized as playing a role in angiogenesis, a histological characteristic that allows for differentiation from simple peritoneal fibrosis to EPS [14].

The endothelial system is another known factor with potent profibrotic characteristics and plays a role in the development of peritoneal fibrosis. This system can be activated by two receptors, endothelial receptors $\mathrm{A}$ and $\mathrm{B}$. However, endothelial receptor B apparently does not play a role in peritoneal membrane thickening in experimental studies inducing deficiency of endothelial receptor B.

Finally, and of extreme importance, infectious peritonitis is an obvious cause of peritoneal inflammation and is associated with EPS development. Gram-positive organisms remain as the more prevalent peritonitis agents over the past decades representing up to $60 \%$ of cases followed by gram-negative organisms. However, the prevalence of peritonitis due gram-negative organisms is growing fast with the development of efficient strategies to control grampositive infections. Despite all efforts made over the past decades, it still represents the most important cause of treatment discontinuation.

In sum, all the above-mentioned factors contribute to the release of proinflammatory cytokines such as interleukin $1 \beta$ (IL $1 \beta$ ), tumor necrosis factor (TNF- $\alpha$ ), IL-6, and IL-18. Structural lesions as a result of this process will be addressed below.

\section{Structural Consequences of Inflammation of PD}

Peritoneal inflammation generated by PD causes significant structural alterations in the peritoneum. These alterations, when severe, can trigger encapsulant peritoneal sclerosis [12]. Mesothelial exposure to PD solution in rats increased cytoplasm in these cells [15]. Thickening and cubic transformation of mesothelial cells occurs and is more accentuated in the parietal peritoneum [16]. Human peritoneal mesothelial cells (HPMCs) also suffer structural alterations and prominent transdifferentiation of HPMC to myofibroblasts occurs [17].

Histological alterations of the peritoneal membrane observed in EPS cases are nonspecific and are masked by the alterations commonly observed in patients with ultrafiltration failure and infectious peritonitis over the long term [18]. The most common findings are fibrin deposition, fibrous capsule formation, perivascular bleeding, interstitial fibrosis, and the presence of tissue granulation with vascular proliferation. Submesothelial tissue thickening also occurs with an increase in deposition of mesothelial conjunctive tissue $[19,20]$. Fibrosis is characterized by the accumulation of extracellular matrix (ECM), resulting in disequilibrium between synthesis and degradation. Expression of collagen types 1 and 3 is significantly increased [21] as well as collagen type 4 [10]. Mesothelial cell denudation has also been described [22]. With respect to neoangiogenisis, we observed an arteriole diabetiform alteration and subendothelial hyalinosis of the venules [23].

\section{Functional Consequence of Inflammation in PD}

Structural alterations of the peritoneal membrane described above result in clinical and functional changes. One of these clinical manifestations is ultrafiltration (UF) failure and can occur in up to $30 \%$ of patients on PD after five years of treatment [1]. One of the presentations of UF failure occurs due to the increase in pores in the peritoneal membrane, which in turn accelerates small-solute transport dissipating the osmotic gradient necessary to maintain adequate fluid balance. This increase in vascular surface is observed in conjunction with an increase in density of interstitial fibers. These findings help justify the increase in transport of small molecules, while the alterations in the UF coefficient are only moderate [24]. In addition to UF failure, clinical manifestations such as severe malnutrition, subocclusion or intestinal occlusion, and ascites suggest the presence of EPS even after discontinuation of PD.

Prescribing more hypertonic glucose solutions is a common strategy to counter this drop in UF, primarily where there is no available icodextrin. This intensifies and perpetuates inflammatory disturbances, with a direct impact on dialysis adequacy and fluid balance. The final consequence is the inevitable transfer to HD. Despite all damage to the peritoneal membrane with therapies performed today, large observational studies have shown an important evolution in PD patient survival when compared to $\mathrm{HD}$ over the past years [25].

\section{Conclusion}

PD initiation increases inflammatory stimuli for the chronic kidney patient such as the presence of the peritoneal catheter, use of bioincompatible solutions, and possible infectious peritonitis. Together, these factors generate structural and physiological alterations of the peritoneal membrane. These manifestations are frequently observed and can range from difficulties in obtaining an adequate fluid balance until the dreaded encapsulant peritoneal sclerosis. Nevertheless, patient survival in $\mathrm{PD}$ is similar to that of HD. An understanding of the mechanisms involved in peritoneal inflammation is fundamental for the development of new strategies. This knowledge can provide not only a better technique survival, but also improvements in patient survival and a better quality of life. 


\section{References}

[1] O. Heimburger, J. Waniewski, A. Werynski, A. Tranaeus, and B. Lindholm, "Peritoneal transport in CAPD patients with permanent loss of ultrafiltration capacity," Kidney International, vol. 38, no. 3, pp. 495-506, 1990.

[2] Y. Kawaguchi, H. Kawanishi, S. Mujais, N. Topley, and D. G. Oreopoulos, "Encapsulating peritoneal sclerosis: definition, etiology, diagnosis, and treatment. International Society for Peritoneal Dialysis Ad Hoc Committee on Ultrafiltration Management in Peritoneal Dialysis," Peritoneal Dialysis International, vol. 20, supplement 4, pp. S43-S55, 2000.

[3] E. A. Brown, W. van Biesen, F. O. Finkelstein et al., "Length of time on peritoneal dialysis and encapsulating peritoneal sclerosis: position paper for ISPD," Peritoneal Dialysis International, vol. 29, no. 6, pp. 595-600, 2009.

[4] A. S. De Vriese, "The John F. Maher recipient lecture 2004: rage in the peritoneum," Peritoneal Dialysis International, vol. 25, no. 1, pp. 8-11, 2005.

[5] M. F. Flessner, K. Credit, K. Henderson et al., "Peritoneal changes after exposure to sterile solutions by catheter," Journal of the American Society of Nephrology, vol. 18, no. 8, pp. 22942302, 2007.

[6] R. T. Krediet, "Effects of icodextrin on the peritoneal membrane," Nephrology Dialysis Transplantation, vol. 25, no. 5, pp. 1373-1375, 2010.

[7] M. R. Korte, D. E. Sampimon, H. F. Lingsma et al., "Risk factors associated with encapsulating peritoneal sclerosis in Dutch eps study," Peritoneal Dialysis International, vol. 31, no. 3, pp. 269-278, 2011.

[8] C. Higuchi, H. Nishimura, and T. Sanaka, "Biocompatibility of peritoneal dialysis fluid and influence of compositions on peritoneal fibrosis," Therapeutic Apheresis and Dialysis, vol. 10, no. 4, pp. 372-379, 2006.

[9] H. Kawanishi, H. Fukui, S. Hara et al., "Encapsulating peritoneal sclerosis in Japan: prospective multicenter controlled study," Peritoneal Dialysis International, vol. 21, supplement 3, pp. S67-S71, 2001.

[10] M. A. M. Mateijsen, A. C. Van Der Wal, P. M. E. M. Hendriks et al., "Vascular and interstitial changes in the peritoneum of CAPD patients with peritoneal sclerosis," Peritoneal Dialysis International, vol. 19, no. 6, pp. 517-525, 1999.

[11] P. Kalk, M. Rückert, M. Godes et al., "Does endothelin B receptor deficiency ameliorate the induction of peritoneal fibrosis in experimental peritoneal dialysis?" Nephrology Dialysis Transplantation, vol. 25, no. 5, pp. 1474-1478, 2010.

[12] K. Kaneko, C. Hamada, and Y. Tomino, "Peritoneal fibrosis intervention,” Peritoneal Dialysis International, vol. 27, supplement 2, pp. S82-S86, 2007.

[13] Q. Yao, K. Pawlaczyk, E. R. Ayala et al., "The role of the TGF/Smad signaling pathway in peritoneal fibrosis induced by peritoneal dialysis solutions," Nephron, vol. 109, no. 2, pp. e71-e78, 2008.

[14] C. Pollock, "Pathogenesis of peritoneal sclerosis," International Journal of Artificial Organs, vol. 28, no. 2, pp. 90-96, 2005.

[15] L. Gotloib and A. Shostak, "Hipertrofia de células mesoteliais inducida por soluciones de diálisis peritonial," Seminários de Nefrologia, vol. 9, article 2, 2006.

[16] P. Vicente, J. Caramori, S. Franco, E. Vicente, J. J. Pereira, and S. Santos, "Efeito da glicose na histomorfologia do peritônio durante a diálise peritoneal," HU Revista, vol. 34, no. 1, pp. 27-31, 2008.
[17] A. S. De Vriese, R. G. Tilton, S. Mortier, and N. H. Lameire, "Myofibroblast transdifferentiation of mesothelial cells is mediated by RAGE and contributes to peritoneal fibrosis in uraemia," Nephrology Dialysis Transplantation, vol. 21, no. 9, pp. 2549-2555, 2006.

[18] K. Honda and H. Oda, "Pathology of encapsulating peritoneal sclerosis," Peritoneal Dialysis International, vol. 25, no. 4, pp. S19-S29, 2005.

[19] K. Y. Hung, J. W. Huang, C. K. Chiang, and T. J. Tsai, "Preservation of peritoneal morphology and function by pentoxifylline in a rat model of peritoneal dialysis: molecular studies," Nephrology Dialysis Transplantation, vol. 23, no. 12, pp. 3831-3840, 2008.

[20] K. Honda, K. Nitta, S. Horita et al., "Histologic criteria for diagnosing encapsulating peritoneal sclerosis in continuous ambulatory peritoneal dialysis patients," Advances in Peritoneal Dialysis, vol. 19, pp. 169-175, 2003.

[21] J. J. Kim, J. J. Li, K. S. Kim et al., "High glucose decreases collagenase expression and increases TIMP expression in cultured human peritoneal mesothelial cells," Nephrology Dialysis Transplantation, vol. 23, no. 2, pp. 534-541, 2008.

[22] D. Bozkurt, P. Cetin, S. Sipahi et al., "The effects of reninangiotensin system inhibition of regression of encapsulation peritoneal sclerosis," Peritoneal Dialysis International, vol. 28, supplement 5, pp. S38-S42, 2008.

[23] R. T. Krediet, A. M. Coester, I. Kolesnyk et al., "Karl D. Nolph state of the art lecture: feasible and future options for salvation of the peritoneal membrane," Peritoneal Dialysis International, vol. 29, supplement 2, pp. S195-S197, 2009.

[24] B. Rippe and D. Venturoli, "Simulations of osmotic ultrafiltration failure in CAPD using a serial three-pore membrane/fiber matrix model," American Journal of Physiology, vol. 292, no. 3, pp. F1035-F1043, 2007.

[25] R. Mehrotra, Y.-W. Chiu, K. Kalantar-Zadeh, J. Bargman, and E. Vonesh, "Similar outcomes with hemodialysis and peritoneal dialysis in patients with end-stage renal disease," Archives of Internal Medicine, vol. 171, no. 2, pp. 110-118, 2011. 


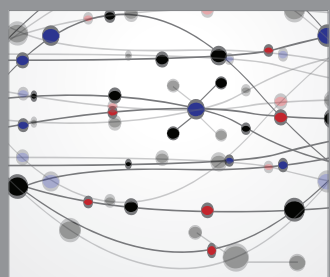

The Scientific World Journal
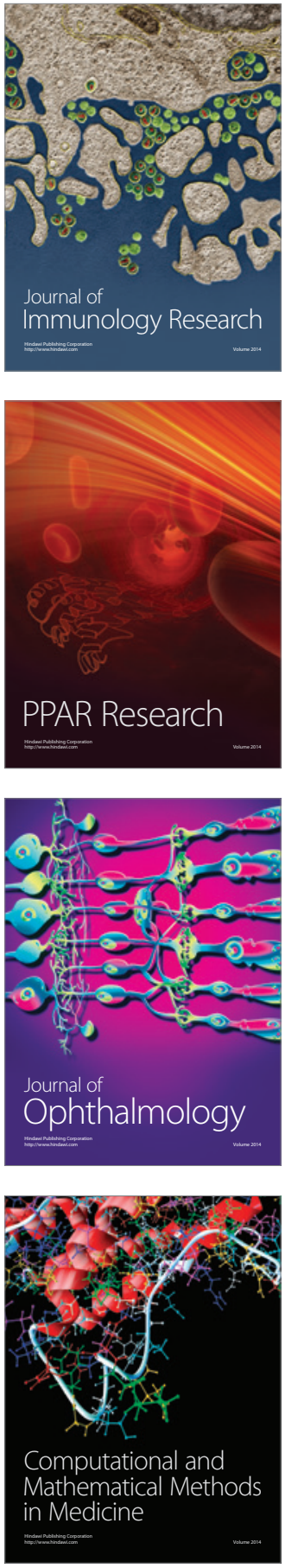

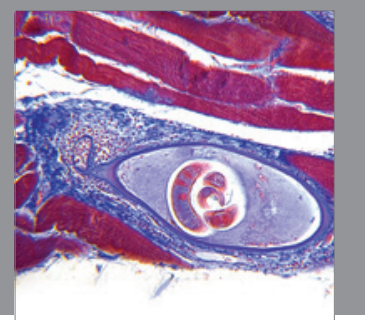

Gastroenterology

Research and Practice
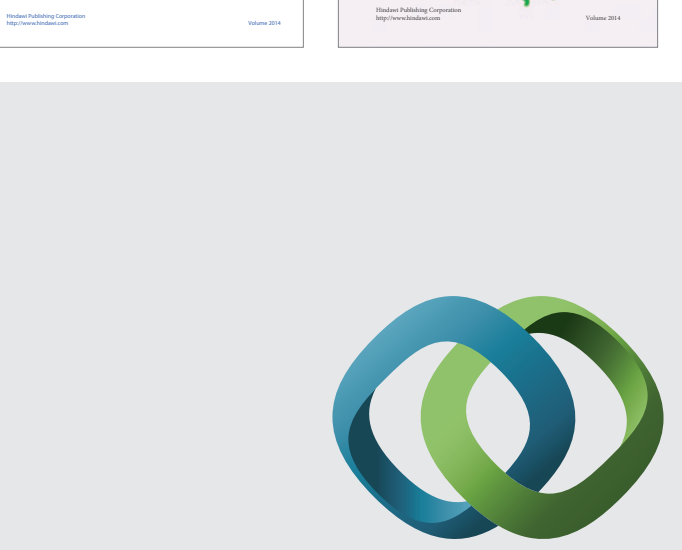

\section{Hindawi}

Submit your manuscripts at

http://www.hindawi.com
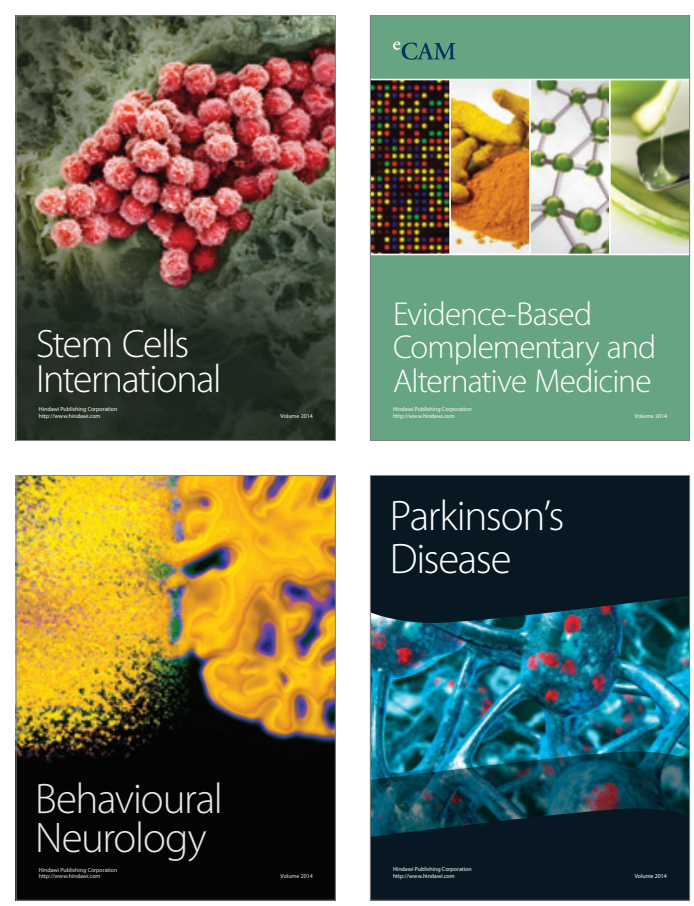

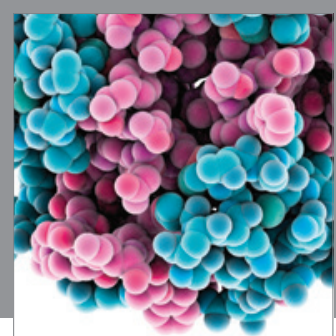

Journal of
Diabetes Research

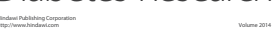

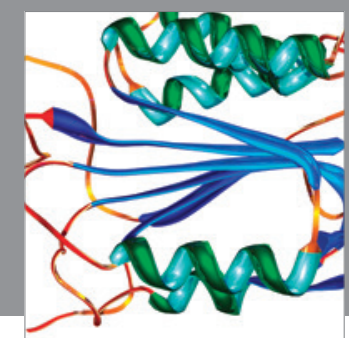

Disease Markers
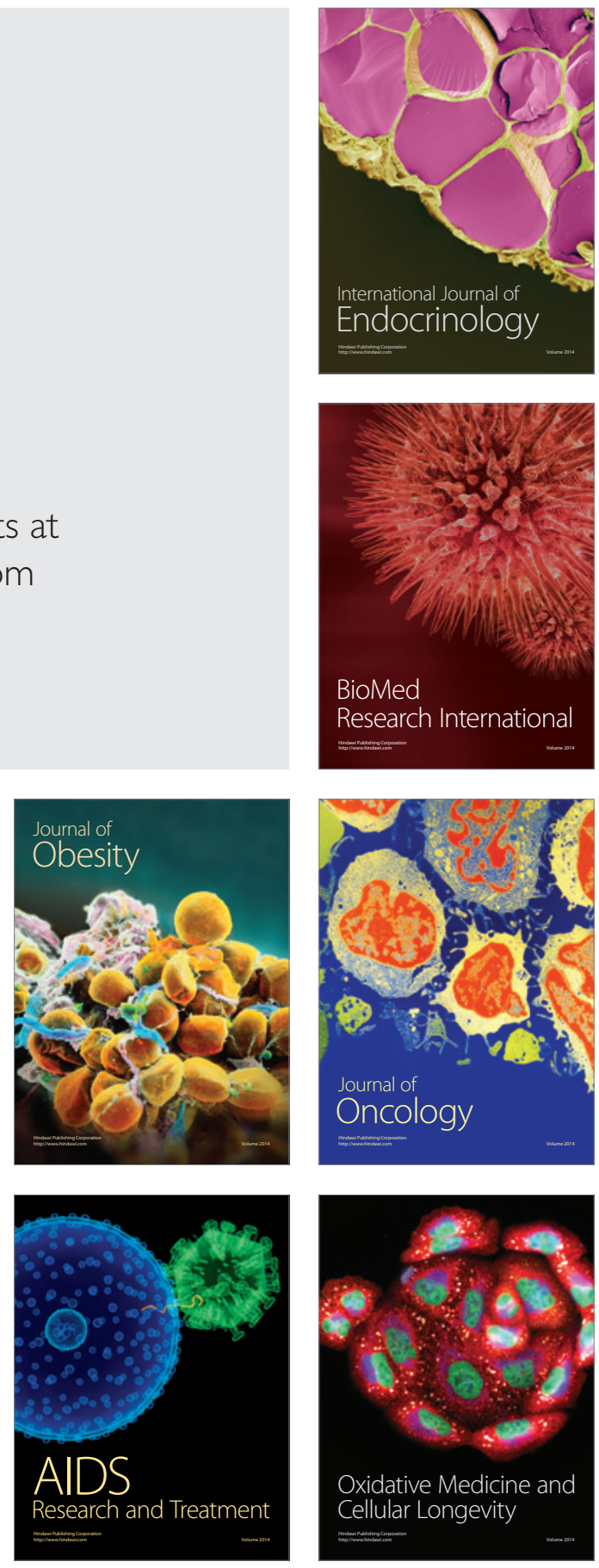